\title{
Hepcidin, Anemia, and Liver Diseases
}

\author{
Shafia Arshad ${ }^{1,2 *}$ and Amina Arif ${ }^{2}$ \\ ${ }^{1}$ Faculty of Medicine and Allied Health Sciences, Islamia University Bahawalpur, Pakistan \\ +Faculty of Life Sciences, University of Central Punjab, Lahore, Pakistan
}

*Corresponding author: Shafia Arshad, Faculty of Medicine and Allied Health Sciences, Islamia University Bahawalpur, Faculty of

Life Sciences, University of Central Punjab, Lahore, Pakistan

\begin{tabular}{|c|c|}
\hline ARTICLE INFO & ABSTRACT \\
\hline Received: 幽 November 17, 2021 & $\begin{array}{l}\text { Citation: Shafia Arshad, Amina Arif. Hepcidin, Anemia, and Liver Diseases. Biomed J Sci } \\
\text { \& Tech Res 40(2)-2021. BJSTR. MS.ID.006422. }\end{array}$ \\
\hline
\end{tabular}

\section{Mini Review}

Hepcidin was found as a tiny bactericidal peptide in human blood and urine called a liver-expressed antimicrobial peptide (LEAP). Hepcidin acts as a homeostatic regulator of systemic iron metabolism as well as a host defense mediator. The liver, which is the prominent location of hepcidin synthesis and secretion, is considered to sense circulating iron and iron storage [1]. Soul of this article explores hepcidin relation with anemia and liver diseases regarding the latest work published. Hepcidin, synthesis is primarily influenced by bone marrow erythropoietic activity, the quantity of circulating and stored body iron, and inflammation [2]. Increased and decreased hepcidin levels are highlighted in many diseases, but the highest lighting is iron deficiency and related disorders. Iron deficiency is the etiology of a disease cluster. Iron deficiency and anemia can be diagnosed by estimation of hepcidin as a diagnostic marker [3,4]. Anemia can be divided into two types based on their hepcidin levels: anemias with high hepcidin and low hepcidin levels. Intuitively, chronically elevated hepcidin levels induce iron deficiency anemia by inhibiting iron absorption and reducing iron bioavailability to erythropoiesis. In contrast, ironloading anemias, which have low hepcidin levels and iron overload, are characterized by inefficient erythropoiesis [5]. In few pieces of research, it is published that opposing hepcidin deregulation may cure anemia in preclinical animals providing novel strategies that are now or will soon be investigated for the treatment of certain anemias [6,7].
Infection, inflammation, erythropoiesis, and hypoxia all affect hepcidin levels. Inflammation and infection raise hepcidin levels, but hypoxia and erythropoiesis lower its level. Anemia of chronic illness is most likely caused by unopposed hepcidin production due to continuous chronic inflammation [8]. Anemia of inflammation and low blood hepcidin levels were discovered to coexist in chronic liver disease patients. In these individuals, the expression of duodenal proteins involved in iron absorption was either reduced or unchanged. Despite liver illness, the hepcidin response to increased body iron levels or inflammation appeared to remain functioning in these individuals [9]. Hepcidin levels have previously been associated with liver injury. In nonalcoholic fatty liver disease (NAFLD), there was a significant inverse correlation between hepcidin, immunoreactivity, and fibrosis. However, serum hepcidin levels were significantly higher, implying that these patients experienced a reduction in the liver's hepcidin-producing ability. In response to above iron levels lead to subsequent fibrosis. As a result, hepcidin levels can be utilized as markers to track the development of fibrosis in NAFLD patients [10]. Hakan, et al. did a study to see if there was a link between blood hepcidin levels at the fibrosis stage and serum iron parameters in individuals with chronic hepatitis B (CHB). They discovered that decreased hepcidin levels and elevated ferritin and transferrin saturation level were linked to the severity of fibrosis [11].

The published scientific data stamps that plasma hepcidin measures may help detect iron-related problems. The therapeutic 
targeting of the hepcidin-ferroportin system is a potential new approach for developing better iron disorders therapies [12]. Different types of chronic liver damage are linked with lower hepcidin mRNA levels, although the consequences on iron status vary. More excellent knowledge of the interplay between diverse stimuli, both positive and negative, on hepcidin regulation is an essential research focus, elucidating the reasons for the differential impact of different chronic injuries on iron homeostasis [13]. Future research should offer a clearer understanding of how hepcidin expression dysregulation and altered iron homeostasis affect the course of liver illnesses and whether they are a cause or a result of these pathologies. The recent invention of assays for measuring hepcidin in serum and urine has opened up new avenues for research into hepcidin regulation in the human body. The use of these assay in diagnosing and medical treatment will ease in eradicating health problems. On-going human research should provide us with additional knowledge on the genesis of iron metabolism disorders, allowing us to develop novel treatment methods. Our understanding of hepcidin's molecular and cellular biology will enable rational treatments that use agonists and antagonists of hepcidin activity, and such drugs are currently being investigated in clinical trials. Extensive, homogenous cohort studies are needed to address connections between iron overload, liver enzymes, and liver function and investigate the potential value of hepcidin in monitoring fibrosis development, liver disorders, and anemia.

\section{References}

1. Hassan IS (2018) To Study of Hepcidin Levels and Certain Hematological Parameters in Pregnant Women. Al-Mustansiriyah J Sci 28(3): 44.

\section{ISSN: 2574-1241}

DOI: 10.26717/BJSTR.2021.40.006422

Shafia Arshad. Biomed J Sci \& Tech Res

This work is licensed under Creative Commons Attribution 4.0 License

Submission Link: https://biomedres.us/submit-manuscript.php
2. Daniel $\mathrm{N}$ Srole, $\mathrm{T}$ Ganz. Erythroferrone structure, function, and physiology: Iron homeostasis and beyond. J Cell Physiol.

3. Camaschella C (2019) Iron deficiency. Blood 133(1): 30-9.

4. Zaman B, Rasool S, Jasim S, Abdulah D (2021) Hepcidin as a diagnostic biomarker of iron deficiency anemia during pregnancy. J Matern Neonatal Med 34(8): 1288-1296.

5. Pagani A, Nai A, Silvestri L, Camaschella C (2019) Hepcidin and Anemia: A Tight Relationship. Front Physiol 10: 1-7.

6. Gambia T, Bah A, Pasricha S, Jallow MW, Sise EA, et al. (2017) Serum Hepcidin Concentrations Decline during Pregnancy and May Identify Iron Deficiency: Analysis of a Longitudinal Pregnancy Cohort in The Gambia. J Nutr 147(6): 1131-1137.

7. Bah A, Muhammad AK, Wegmuller R, Verhoef H, Goheen MM, et al. (2019) Hepcidin-guided screen-and-treat interventions against irondeficiency anaemia in pregnancy: a randomised controlled trial in The Gambia. Lancet Glob Heal 7(11): 1564-74.

8. Kowdley K V, Gochanour EM, Sundaram V, Shah RA, Handa P (2021) Hepcidin Signaling in Health and Disease: Ironing Out the Details. Hepatol Commun 5(5): 723-35.

9. Varghese J, Varghese James J, Karthikeyan M, Rasalkar K, Raghavan R, et al. (2020) Iron homeostasis is dysregulated, but the iron-hepcidin axis is functional, in chronic liver disease. J Trace Elem Med Biol 58: 126442.

10. Norito Tsutsumi, Shigeo Nishimata, Masaru Shimura, Yasuyo Kashiwagi, Hisashi Kawashima (2021) Hepcidin Levels and Pathological Characteristics in Children with Fatty Liver Disease. Pediatr Gastroenterol Hepatol Nutr 24(3): 295-305.

11. Çam H, Yllmaz N (2020) Serum hepcidin levels are related to serum markers for iron metabolism and fibrosis stage in patients with chronic hepatitis B: A cross-sectional study. Arab J Gastroenterol 21(2): 85-90.

12. Ganz EN, Tomas (2021) Hepcidin-Ferroportin Interaction Controls Systemic Iron Homeostasis. Int J Mol Sci 22(12): 6493.

13. Bloomer SA, Brown KE (2021) Hepcidin and Iron Metabolism in Experimental Liver Injury. Am J Pathol 191(7): 1165-79.

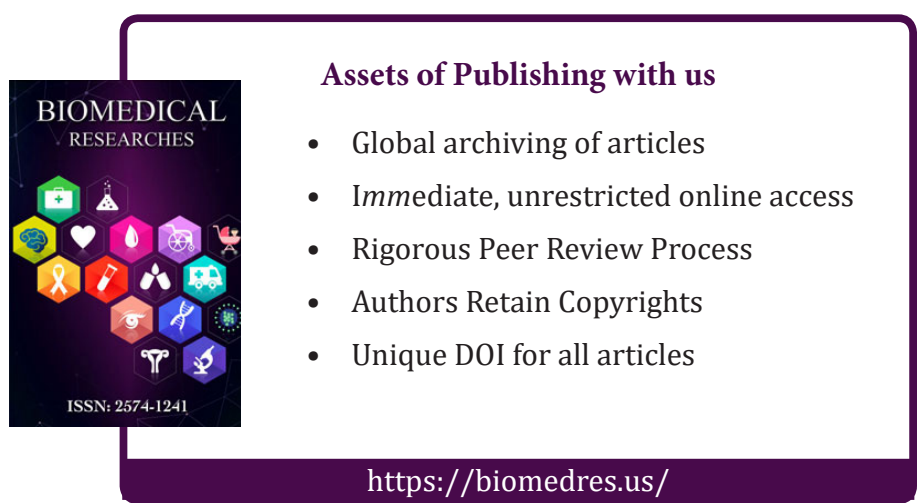

